\title{
CD31-positive microvessel density within adenomas of Lynch Syndrome patients is similar compared to adenomas of non-Lynch patients
}

(๑) $\odot \ominus$

\author{
Authors \\ Jasper L.A. Vleugels ${ }^{1,}{ }^{*}$, Sanne M. van Neerven ${ }^{2,}{ }^{*}$, Monique E. van Leerdam³, Linda K. Wanders' ${ }^{1}$, Meike de Wit ${ }^{4}$, \\ Beatriz Carvalho ${ }^{4}$, Pien M. Delis-van Diemen ${ }^{4}$, Frank G.J. Kallenberg ${ }^{1}$, Louis Vermeulen², Jeroen A. Beliën ${ }^{4,5}$, \\ James E. East ${ }^{6}$, Gerrit A. Meijer ${ }^{4}$, Evelien Dekker ${ }^{1}$
}

Institutions

1 Department of Gastroenterology and Hepatology, Amsterdam University Medical Center, location Academic Medical Center, Amsterdam, the Netherlands

2 Cancer Center Amsterdam, Laboratory for Experimental Oncology and Radiobiology (LEXOR), Center for Experimental and Molecular Medicine (CEMM), Amsterdam University Medical Center, location Academic Medical Center, Amsterdam, the Netherlands

3 Department of Gastroenterology and Hepatology, Netherlands Cancer Institute, Amsterdam, the Netherlands

4 Department of Pathology, Netherlands Cancer Institute, Amsterdam, the Netherlands

5 Department of Pathology, Amsterdam University Medical Center, location VU University Medical Centre, Amsterdam, the Netherlands

6 Translational Gastroenterology Unit, Nuffield Department of Medicine, University of Oxford, United Kingdom

submitted 25.9.2018

accepted after revision 4.12 .2018

Bibliography

DOI https://doi.org/10.1055/a-0832-8283 |

Endoscopy International Open 2019; 07: E701-E707

(c) Georg Thieme Verlag KG Stuttgart · New York eISSN 2196-9736

Corresponding author

Professor Evelien Dekker, MD PhD, Department of Gastroenterology and Hepatology, Amsterdam University Medical Center, location Academic Medical Center, Meibergdreef 9, 1105 AZ, Amsterdam, the Netherlands Fax: +31206917033

e.dekker@amc.uva.nl

\section{ABSTRACT}

Background and study aims Microsatellite instability accelerates colorectal cancer development in patients with Lynch syndrome (LS). Previous research showed that virtual chromoendoscopy increases detection of adenomas during colonoscopy surveillance of patients with LS. Because previous research revealed that Lynch patients have an increased vascular network in the oral mucosa, we hypothesized that increased vascularization of LS-associated adenomas is the cause of better detection with virtual chromoendoscopy.

Patients and methods In this pilot study, patients with LS having a proven germline mutation were selected from two tertiary referral hospitals and non-LS patients from an outpatient colonoscopy center. Adenomas from patients with LS were exactly matched in size and histology with adenomas from non-LS patients. Initial adenoma diagnosis was confirmed by a specialist pathologist. All adenomas were stained with CD31 and adenomatous tissue was annotated by the specialist pathologist. Image analysis of CD31-positive microvessel density was conducted using FIJI software. Results Colonoscopy of 63 patients with LS and 24 non-LS patients provided 40 adenomas that could be exactly matched in size and histology. In image-analysis, the CD31-positive microvessel density $(2.49 \%$ vs. $2.47 \%, P=0.96)$, the average size of CD31-positive structures $\left(514 \mu \mathrm{m}^{2}\right.$ vs. $\left.523 \mu \mathrm{m}^{2}, P=0.26\right)$ nor the amount of vascular structures per $\mathrm{mm}^{2}$ ( 183 vs. $176, P=0.50$ ) differed between adenomas of LS patients and non-Lynch patients.

Conclusion The outcomes of this pilot case-control study did not provide further insights into the mechanism of increased adenoma detection in LS patients using virtual chromoendoscopy techniques.

* These authors contributed equally. 


\section{Introduction}

Lynch syndrome (LS) is a hereditary disorder caused by a mutation in a mismatch repair (MMR) gene [1]. Affected individuals have a high chance of developing colorectal cancer (CRC), often at a young age $[1,2]$. To prevent CRC by detecting and removing precursor lesions, patients with LS are currently advised to undergo colonoscopy every 1 to 2 years beginning at age 25 [3-5]. However, despite strict colonoscopy surveillance, patients with LS still develop post-colonoscopy CRCs [6-8]. The two most commonly cited reasons are the accelerated adenoma-carcinoma pathway due to microsatellite instability [9], and potentially missed lesions during previous colonoscopy $[8,10]$. Therefore, colonoscopic inspection should be very meticulous and precise in these patients.

To improve lesion detection during colonoscopy, several advanced imaging techniques have been studied in patients with LS [11 - 17]. Besides traditional chromoendoscopy [11-15], in which topical application of dye is used, these include virtual chromoendoscopy techniques as narrow band imaging (NBI) and i-Scan [16, 17]. In NBI, emitted light passes through a special narrow-band, eliminating all other wavelengths except for two specific wavelengths $(415 \mathrm{~nm}$ and $540 \mathrm{~nm}$ ) that are strongly absorbed by hemoglobin. As a result, the contrast of the blood vessels in relation to the mucosa is enhanced. i-Scan consists of post-processing light filter technology that uses software algorithms to enhance different mucosal and vascular features. Because adenomas have increased vascularity compared to normal mucosa, adenomatous tissue appears brown due to this increased vessel density, against a green-blue background. Use of these virtual chromoendoscopy techniques may enhance detection of adenomas during colonoscopy.

Based on two studies in patients with LS $[16,17]$, the European Society of Gastrointestinal Endoscopy (ESGE) suggests routine use of virtual chromoendoscopy (NBI, i-Scan) in patients with LS [18]. However, virtual chromoendoscopy techniques have not proven to be beneficial for improving adenoma detection in the average-risk population [19-21]. Previous research has shown that patients with LS have an increased vascular network complexity in the mouth compared to non-LS carriers [22]. Possibly, this may also be true for the vascular network in LS-associated adenomas. As virtual chromoendoscopy techniques highlight vascular structures, increased adenoma detection using virtual chromoendoscopy could be explained by increased vascularization of Lynch-associated adenomas compared to non-Lynch adenomas.

In this study, we tested this hypothesis by comparing CD31positive microvessel density between LS-associated adenomas and adenomas from non-LS patients, i. e. sporadic adenomas.

\section{Patients and methods}

\section{Study design}

This study was designed as a case-control pilot study in the Academic Medical Center (AMC), Amsterdam, and the Antoni van Leeuwenhoek-Netherlands Cancer Institute (AvL-NKI), Amsterdam. These are two tertiary referral centers providing integra- ted care for patients with LS. The local Institutional Review Boards of the AMC declared that this study did not require any formal approval as data were collected during standard care. Collection, storage, and use of tissue specimens was performed according to the prevailing guideline "Code for proper secondary use of human tissue" in the Netherlands. All data and tissue were handled anonymously throughout the study.

\section{Matching of cases and controls}

This study was merely performed as a feasibility study without a formal sample size calculation. To our knowledge, this is the first study comparing CD31-positive microvessel density of adenomas and no estimates could be derived from literature to perform a valid sample size calculation. We decided to match 20 adenomas of patients with LS 1:1 with 20 sporadic adenomas on endoscopic adenoma size and histology. The rationale was that increasing adenoma size and histology could possibly influence the vascularization of adenomas.

If a patient provided multiple adenomas for matching, only one adenoma per patient was included for this analysis to exclude the possibility that vascularization of adenomas depended on patient characteristics. If the quality or amount of adenomatous tissue within paraffin-embedded sample for CD31 staining was considered insufficient, matching was redone until 20 representative cases and 20 representative controls were selected.

\section{Selection of cases and controls}

LS patients were selected from two registries in these two tertiary referral centers when at least one adenoma was resected during surveillance. Only patients diagnosed with LS by a proven germline mutation in one of the four mismatch repair genes (MLH1, MSH2, MSH6 or PMS2) were included in this study. Patients diagnosed with a concomitant polyposis syndrome or inflammatory bowel disease, or aged younger than 18 at time of colonoscopy were not included in this study.

Non-LS patients were selected from an outpatient colonoscopy center. In this center, the majority of colonoscopies was performed for a positive fecal immunochemical test (FIT), symptoms or surveillance for earlier detected polyps. This cohort has been described in detail previously [23]. Patients were selected if an adenoma was resected. Endoscopies in patients with previously diagnosed inflammatory bowel disease, LS or polyposis syndrome were not included. All patients undergoing colonoscopy in this outpatient clinic were required to fill in a questionnaire that included a precise description of CRC family history. To rule out the possibility that these patients were at risk for LS, we included only those patients who did not meet clinical criteria for Lynch testing (revised Bethesda or Amsterdam II criteria) or familial colorectal cancer (FCC) to serve as controls in this study [5, 24].

For all patients, individual medical history was retrieved, including colonoscopy information and histopathology reports. Patient demographics such as age and gender were recorded and we retrieved the findings per colonoscopy. Per detected lesion, the anatomic location, endoscopic size, Paris morphology and histology outcome were recorded. 


\section{Histopathology review}

All matched adenomatous lesions were reviewed by a specialist gastrointestinal pathologist (GAM) according to World Health Organization 2010 pathology guidelines to confirm the initial diagnosis [25].

\section{Material preparation}

Formalin-fixed, paraffin-embedded samples of histologically proven adenomas of cases and controls removed during colonoscopy between 2007 and 2015 were retrieved. Four-micrometer sections of histologically confirmed adenomas were mounted on glass slides after deparaffinization and rehydration. These tissue sections were treated with $10 \mathrm{mM}$ citrate buffer $(\mathrm{pH} 6)$ in a microwave for 5 minutes at maximum power followed by 10 minutes at 360 watts for the CD31 staining. Hereafter, sections were incubated at room temperature with CD31 antibody. Signals were visualized with horse-radish peroxidase.

\section{Image analysis}

Prior to image analysis, the specialist gastrointestinal pathologist (GAM) manually annotated the adenomatous tissue regions visible in the CD31-stained slides. Consequently, only adenomatous tissue was included in image analysis of CD31positive microvessel density. An analysis of the adenomatous tissue was performed using FIJl software where hematoxylin and DAB stainings were separated using color deconvolution [26]. Next, the binary images were thresholded equally prior to measuring the area of CD31-positive particles. Conditions for CD31 detection were set to be minimally $100 \mu \mathrm{m}^{2}$ with a circularity of 0.05 to exclude CD31-positive lymphocytes and staining artifacts ( $\triangleright$ Fig. 1 ). For measuring the area of hematoxylin staining, no further conditions were set, and all particles were included in the analysis.

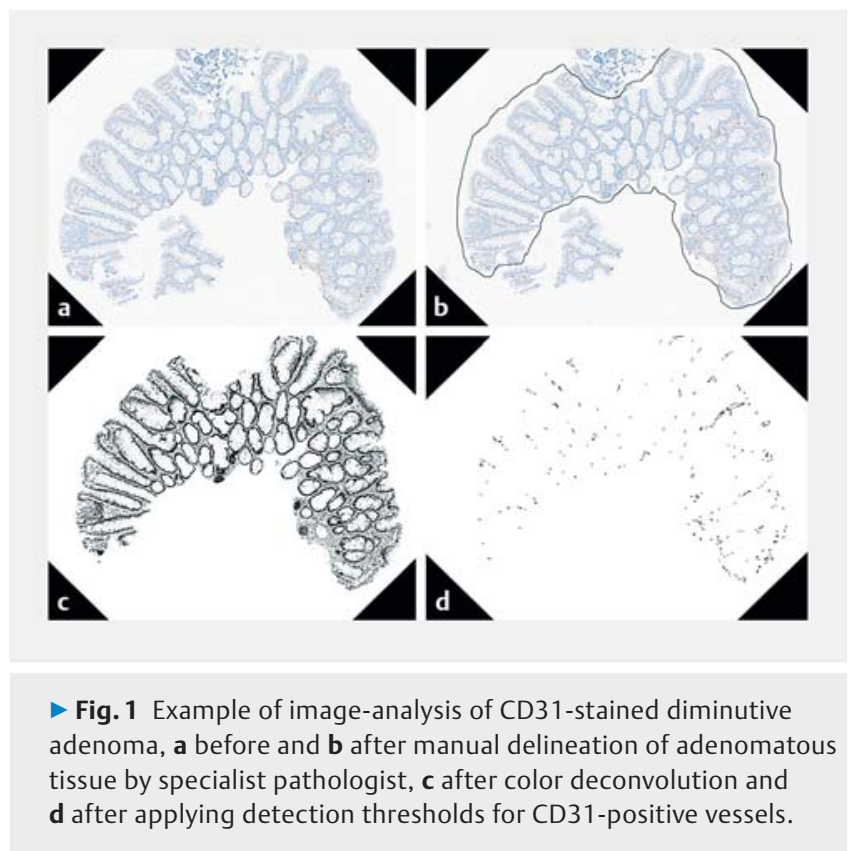

\section{Outcome parameters and statistical analysis}

The main outcome parameter was CD31-positive microvessel density, expressed as the proportion of total surface of CD31positive particles divided by the total area of annotated adenomatous tissue and displayed as mean including the standard deviation (SD). Other main outcome parameters included average size of CD31-positive structures in $\mu \mathrm{m}^{2}$ described as median and interquartile range (IQR), and the amount of CD31-positive structures per $\mathrm{mm}^{2}$ situated within the annotated adenomatous regions described as median and IQR. The main outcome measures were verified for normal distribution and subsequently analyzed using the paired $t$-test and Wilcoxon signed rank test.

Patient characteristics were described using descriptive statistics. Subgroup analysis were performed for male versus female gender, MMR mutation, adenoma location and morphology did not change any results. The proximal colon was defined as proximal to the descending colon. The left-sided colon included the descending colon and all distal parts. Morphology was defined as flat (-elevated) versus non-flat (sessile and pedunculated). To compare differences between the LS-associated adenomas and sporadic adenomas regarding their clinicopathologic features, the Wilcoxon signed rank test, paired $t$ test or McNemar test were used when appropriate. SPSS version 24 (SPSS Statistics for Windows; IBM, Armonk, New York, United States) and GraphPad Prism version 7.03 (GraphPad Software, La Jolla, California, United States) were used for statistical analysis. A $P$ value $<0.05$ was considered to be statistically significant.

\section{Results}

\section{Description of cases and controls}

We selected 63 patients with LS (34 from AvL-NKI, 29 from AMC) and 24 non-LS patients. During colonoscopy surveillance of patients with LS performed between 2007 and 2015, 105 adenomas were selected for matching. Colonoscopy of the 24 non-Lynch patients, performed between 2014 and 2015, provided 23 adenomas for matching. After histopathology review and staining, 38 adenomas of patients with LS and two sporadic adenomas of non-Lynch patients were excluded because the patient provided multiple adenomas for matching $(N=17)$, there was insufficient material for new slide preparation $(N=15)$ or the primary diagnosis could not be confirmed $(\mathrm{N}=8)$.

Twenty adenomas of LS patients were exactly matched to the 20 sporadic adenomas in size and histology ( $\vee$ Table 1 ). All 40 adenomas were tubular adenomas with low-grade dysplasia. For the analyzed adenomas, endoscopic location and morphology did not differ between the two groups. Compared to the non-Lynch patients, Lynch patients had a younger mean age ( $55 \pm 9$ versus $63 \pm 8$ years, $P=0.004$ ). 
- Table 1 Characteristics of patients and adenomas in the Lynch and non-Lynch cohort.

\begin{tabular}{|c|c|c|c|}
\hline & $\begin{array}{l}\text { Lynch } \\
(\mathrm{N}=\mathbf{2 0})\end{array}$ & $\begin{array}{l}\text { Non- } \\
\text { Lynch } \\
(N=20)\end{array}$ & $P$ value \\
\hline \multicolumn{4}{|l|}{ Patient characteristics } \\
\hline Age, mean (SD) & $55( \pm 9)$ & $63( \pm 8)$ & 0.004 \\
\hline Female gender, n (\%) & $55 \%$ & $45 \%$ & 0.72 \\
\hline \multicolumn{4}{|l|}{ MMR mutation, n (\%) } \\
\hline MLH1 & $5(13 \%)$ & - & \\
\hline $\mathrm{MSH} 2$ & $8(20 \%)$ & - & \\
\hline MSH6 & $6(15 \%)$ & - & \\
\hline PMS2 & $1(2 \%)$ & - & \\
\hline \multicolumn{4}{|l|}{ Indication colonoscopy, n (\%) } \\
\hline FIT-positive & - & $10(50 \%)$ & \\
\hline Symptoms & - & $4(20 \%)$ & \\
\hline Surveillance & $20(100 \%)$ & $6(30 \%)$ & \\
\hline \multicolumn{4}{|l|}{ Characteristics of adenomas } \\
\hline \multicolumn{4}{|l|}{ Location, n (\%) } \\
\hline Proximal colon & $10(50 \%)$ & $8(40 \%)$ & 0.48 \\
\hline Distal colon & $10(50 \%)$ & $12(60 \%)$ & \\
\hline $\begin{array}{l}\text { Median endoscopic size in } \\
\mathrm{mm}(\mathrm{IQR})^{*}\end{array}$ & $3(2-4)$ & $3(2-4)$ & 1.00 \\
\hline \multicolumn{4}{|l|}{ Morphology, n (\%) } \\
\hline Sessile & $11(55 \%)$ & $14(70 \%)$ & 0.69 \\
\hline Pedunculated & 0 & $1(5 \%)$ & \\
\hline Flat (elevated) & $9(45 \%)$ & $5(25 \%)$ & \\
\hline \multicolumn{4}{|l|}{ Advanced histology, $\mathrm{n}(\%)^{*}$} \\
\hline$>25 \%$ villous features & 0 & 0 & 1.00 \\
\hline High-grade dysplasia & 0 & 0 & \\
\hline \multicolumn{4}{|c|}{$\begin{array}{l}\text { * Adenomas in this study were matched on endoscopic adenoma size and } \\
\text { adenoma histology. SD, standard deviation; n, number; MMR, mismatch } \\
\text { repair gene; FIT, fecal immunochemical test; IQR, interquartile range }\end{array}$} \\
\hline
\end{tabular}

\section{Main outcome parameters}

CD31-positive microvessel density was $2.49 \%(S D \pm 1.55)$ in adenomas of LS patients compared to $2.47 \%(S D \pm 1.71)$ in sporadic adenomas $(P=0.96)$. The average size of CD31-positive structures did not significantly differ between the two groups: $514 \mu \mathrm{m}^{2}$ (IQR $\left.451-587\right)$ versus $523 \mu \mathrm{m}^{2}$ (IQR $485-$ $605, P=0.26)$. The number of CD31-positive vascular structures per $\mathrm{mm}^{2}$ was also not significant different between the Lynch and sporadic adenomas: 183 (IQR 68-230) versus 176 (IQR $107-262, P=0.50)$. A graphical display of the main outcome measures including all individual results is shown in $\mathbf{F i g . 2}$ Subgroup analysis for male gender, MMR mutation, adenoma location, and morphology did not change any results.

\section{Discussion}

In this translational matched case-control study, we compared the CD31-positive microvessel density between 20 LS-associated adenomas and 20 sporadic adenomas removed during colonoscopy. In structured image-analysis, we found no significant differences in CD31-positive microvessel density, average size of CD31-positive vascular structures or number of CD31-positive vascular structures per $\mathrm{mm}^{2}$ between the two groups. Previous studies showed that virtual chromoendoscopy techniques NBI and i-Scan improved adenoma detection in LS patients $[16,17]$, whereas use of these virtual chromoendoscopy techniques did not increase adenoma detection rates in the average-risk population [19-21]. For this study, we hypothesized that improved adenoma detection by virtual chromoendoscopy during surveillance of Lynch patients could be a result of increased vascularity of adenomas in those patients compared with adenomas in the average-risk population, as previous research revealed that Lynch patients have an increased vascular network in the oral mucosa [22]. However, the results of this case-control study reject this hypothesis and do not explain the mechanism of increased adenoma detection in patients with LS using virtual chromoendoscopy.

The two studies mentioned in the European Society of Gastrointestinal Endoscopy guideline are a recently published randomized back-to-back colonoscopy trial by Bisschops et al., where use of i-Scan significantly decreased the adenoma miss rate in patients with LS from $62 \%$ to $12 \%(P=0.007)$ compared to use of high-definition white light (HD-WLE) [16]. Furthermore, the adenoma detection rate using i-Scan was $23 \%$ compared to $13 \%$ for HD-WLE, independent of inspection time. In 2008, East et al. used a similar design, but patients were not randomized for order of techniques: In all patients the proximal colon was first assessed with HD-WLE and subsequently with $\mathrm{NBI}$ [17]. The additional NBI pass significantly increased the number of patients with at least one adenoma (from $27 \%$ to $42 \%$ ), the total number of adenomas (from 25 to 46) and the proportion of flat adenomas (from $12 \%$ to $45 \%$ ). As there seems to be no difference in vascularization between LS-associated and sporadic adenomas, the incremental effect of virtual chromoendoscopy techniques in adenoma detection in patients with LS may be overestimated. The studies of Bisschops et al. and East et al. used a back-to-back colonoscopy design, and were performed by single-center experienced academic endoscopists. Because blinding for the imaging technique is impossible, this may have swayed the endoscopists' towards virtual chromoendoscopy techniques. It therefore remains unclear whether these findings can be corroborated in a parallel colonoscopy trial. Furthermore, patients included in the studies from East et al. and Bisschops et al. were heterogeneous as patients had proven MMR mutations in only $13 \%$ and $64 \%$, respectively, and thus the diagnosis of LS was not confirmed in many. Hence, future multicenter studies evaluating use of virtual chromoendoscopy in genetically confirmed LS patients in general endoscopy practice are needed to corroborate these previous findings. 




- Fig. 2 Main outcome measures of structured image-analysis presented according to matched pairs.

When using virtual chromoendoscopy during colonoscopy, only the outer surface of the lesion can be investigated. In this study, we took the total CD31-positive area from a cross-section of the adenoma. Because included adenomas were already resected and sectioned, it was impossible to retrospectively determine the outer surface of the adenoma. Also, we did not evaluate vascular endothelial growth factor A (VEGF-A) or other vascular markers in this study. VEGF-A has been shown to be overexpressed early in the adenoma-carcinoma sequence due to an angiogenic switch $[27,28]$. In a recently published study, there was moderate to strong VEGF-A expression in the majority of adenomas in LS patients [29]. However, no difference in VEGF-A expression between LS-associated adenomas and sporadic adenomas was detected [29]. VEGF-A expression rates of sporadic adenomas were in concordance with the literature, although no information on the endoscopic size of these lesions was presented [29]. Future studies looking into vascularization of adenomas in LS patients could potentially take VEGF-A and other vascular markers into account.

It is possible that the small sample of cases and controls in this pilot study did not allow for detection of significant differences in CD31-positive microvessel density. Another reason for not finding a difference could be that we compared microvessel density in small adenomas, as the median size was $3 \mathrm{~mm}$ in each group. Because the analyzed adenomas were small, their vascularization may be much less compared to larger lesions, making it more difficult to detect a difference in vascularization between those lesions. Moreover, these lesions were removed by either cold snare or biopsy forceps, and in the majority of lesions, only small areas of adenomatous tissue could be annotated by the specialist pathologist. However, the increase in adenoma detection in the two back-to-back using NBI and i-Scan was mainly based on better detection of diminutive ( $1-5 \mathrm{~mm}$ ) and small $(6-9 \mathrm{~mm})$ adenomas $[16,17]$. Therefore, the adenomas analyzed in this case-control study represent the findings of these studies. Another reason for not finding a difference is that some of the adenomatous regions in LS adenomas also contained normal mucosa, which is known to be less well vascularized compared to adenomas. On the other hand, this would also apply to the sporadic adenoma group. Last, to exclude CD31-positive lymphocytes and staining artifacts in the image analysis, we set minimal requirements for a CD31-positive microvessel in terms of surface and circularity. However, we cannot entirely exclude the possibility that we included some artifacts or CD31-positive lymphocytes and that this may have influenced the results, although we suspect this to be randomly distributed between the two groups.

Traditional dye-spray chromoendoscopy has also proven to improve adenoma detection in LS surveillance [11 - 15]. Therefore, several countries have recommended traditional dyespray chromoendoscopy as a surveillance method for patients with LS. However, this technique is laborious, time-consuming and requires experience. Therefore, virtual chromoendoscopy is an attractive alternative. Ideally, long-term and large-scale follow-up studies would determine whether increasing adenoma detection rates in LS patients also results in reduced CRC incidence and mortality. It is hoped that this could also result in a less strict colonoscopy surveillance protocol and extension of current recommended colonoscopy intervals. 


\section{Conclusion}

Virtual chromoendoscopy techniques can improve adenoma detection by enhancing the contrast between vascular structures and normal mucosa and these techniques have indeed shown to increase detection of adenomas in LS surveillance, but not in the average-risk population. The outcomes of this translational matched case-control study did not confirm our hypothesis that increased detection of adenomas in LS patients by virtual chromoendoscopy techniques was based on increased vascularization in LS compared to sporadic adenomas. Because this study did not confirm our hypothesis, we could not explain the mechanism of increased adenoma detection in patients with LS using virtual chromoendoscopy.

\section{Acknowledgement}

Prof. James East was funded by the National Institute for Health Research (NIHR) Oxford Biomedical Research Centre (BRC). The views expressed are those of the author(s) and not necessarily those of the NHS, the NIHR or the Department of Health.

\section{Competing interests}

Dr. van Neerven is supported by a NOW PhD scholarship (022.005.002). Dr. Dekker has equipment on loan and receives a consultancy-fee and unrestricted research grant from FujiFilm, has equipment on loan and receives a consultancy-fee from Olympus, and receives a consultancy fee from Tillots.

\section{References}

[1] Lynch HT, de la Chapelle A. Hereditary colorectal cancer. N Engl J Med 2003; 348: 919-932

[2] Jarvinen HJ, Aarnio M, Mustonen $\mathrm{H}$ et al. Controlled 15 -year trial on screening for colorectal cancer in families with hereditary nonpolyposis colorectal cancer. Gastroenterology 2000; 118: 829-834

[3] Giardiello FM, Allen JI, Axilbund JE et al. Guidelines on genetic evaluation and management of Lynch syndrome: a consensus statement by the US Multi-society Task Force on colorectal cancer. Am J Gastroenterol 2014; 109: 1159-1179

[4] Vasen HF, Blanco I, Aktan-Collan K et al. Revised guidelines for the clinical management of Lynch syndrome (HNPCC): recommendations by a group of European experts. Gut 2013; 62: 812-823

[5] Stoffel EM, Mangu PB, Gruber SB et al. Hereditary colorectal cancer syndromes: American Society of Clinical Oncology Clinical Practice Guideline endorsement of the familial risk-colorectal cancer: European Society for Medical Oncology Clinical Practice Guidelines. J Clin Oncol 2015; 33: 209-217

[6] Dove-Edwin I, Sasieni P, Adams J et al. Prevention of colorectal cancer by colonoscopic surveillance in individuals with a family history of colorectal cancer: 16 year, prospective, follow-up study. BMJ 2005; 331: 1047

[7] Vasen HF, Abdirahman M, Brohet R et al. One to 2-year surveillance intervals reduce risk of colorectal cancer in families with Lynch syndrome. Gastroenterology 2010; 138: 2300-2306
[8] Haanstra JF, Vasen HF, Sanduleanu S et al. Quality colonoscopy and risk of interval cancer in Lynch syndrome. Int J Colorectal Dis 2013; 28: $1643-1649$

[9] Edelstein DL, Axilbund J, Baxter M et al. Rapid development of colorectal neoplasia in patients with Lynch syndrome. Clin Gastroenterol Hepatol 2011; 9: 340-343

[10] Rondagh EJ, Gulikers S, Gomez-Garcia EB et al. Nonpolypoid colorectal neoplasms: a challenge in endoscopic surveillance of patients with Lynch syndrome. Endoscopy 2013; 45: 257-264

[11] Lecomte T, Cellier C, Meatchi T et al. Chromoendoscopic colonoscopy for detecting preneoplastic lesions in hereditary nonpolyposis colorectal cancer syndrome. Clin Gastroenterol Hepatol 2005; 3: $897-902$

[12] Hurlstone DP, Karajeh M, Cross SS et al. The role of high-magnification-chromoscopic colonoscopy in hereditary nonpolyposis colorectal cancer screening: a prospective "back-to-back" endoscopic study. Am J Gastroenterol 2005; 100: 2167-2173

[13] Stoffel EM, Turgeon DK, Stockwell DH et al. Missed adenomas during colonoscopic surveillance in individuals with Lynch Syndrome (hereditary nonpolyposis colorectal cancer). Cancer Prev Res (Phila) 2008; 1: $470-475$

[14] Huneburg R, Lammert F, Rabe C et al. Chromocolonoscopy detects more adenomas than white light colonoscopy or narrow band imaging colonoscopy in hereditary nonpolyposis colorectal cancer screening. Endoscopy 2009; 41: 316-322

[15] Rahmi G, Lecomte T, Malka D et al. Impact of chromoscopy on adenoma detection in patients with Lynch syndrome: a prospective, multicenter, blinded, tandem colonoscopy study. Am J Gastroenterol 2015; 110: $288-298$

[16] Bisschops R, Tejpar S, Willekens H et al. Virtual chromoendoscopy (ISCAN) detects more polyps in patients with Lynch syndrome: a randomized controlled crossover trial. Endoscopy 2017; 49: 342-350

[17] East JE, Suzuki N, Stavrinidis M et al. Narrow band imaging for colonoscopic surveillance in hereditary non-polyposis colorectal cancer. Gut 2008; 57: $65-70$

[18] Kaminski MF, Hassan C, Bisschops R et al. Advanced imaging for detection and differentiation of colorectal neoplasia: European Society of Gastrointestinal Endoscopy (ESGE) Guideline. Endoscopy 2014; 46: $435-449$

[19] Nagorni A, Bjelakovic G, Petrovic B. Narrow band imaging versus conventional white light colonoscopy for the detection of colorectal polyps. Cochrane Database Syst Rev 2012; 1: Cd008361

[20] Pasha SF, Leighton JA, Das A et al. Comparison of the yield and miss rate of narrow band imaging and white light endoscopy in patients undergoing screening or surveillance colonoscopy: a meta-analysis. Am J Gastroenterol 2012; 107: $363-370$

[21] Hong SN, Choe WH, Lee JH et al. Prospective, randomized, back-toback trial evaluating the usefulness of i-SCAN in screening colonoscopy. Gastrointest Endosc 2012; 75: 1011 - 1021.e2

[22] De Felice C, Latini G, Bianciardi G et al. Abnormal vascular network complexity: a new phenotypic marker in hereditary non-polyposis colorectal cancer syndrome. Gut 2003; 52: 1764 - 1767

[23] IJspeert JE, van Doorn SC, van der Brug YM et al. The proximal serrated polyp detection rate is an easy-to-measure proxy for the detection rate of clinically relevant serrated polyps. Gastrointest Endosc 2015; 82: $870-877$

[24] Umar A, Boland CR, Terdiman JP et al. Revised Bethesda Guidelines for hereditary nonpolyposis colorectal cancer (Lynch syndrome) and microsatellite instability. J Natl Cancer Inst 2004; 96: 261-268

[25] Bosman FTC, Hruban RH, Theise ND. WHO Classification of Tumours of the Digestive System, Fourth Edition. WHO Classification of Tumours 2010: Volume 3 
[26] Schindelin J, Arganda-Carreras I, Frise E et al. Fiji: an open-source platform for biological-image analysis. Nat Methods 2012; 9: 676682

[27] Staton CA, Chetwood AS, Cameron IC et al. The angiogenic switch occurs at the adenoma stage of the adenoma carcinoma sequence in colorectal cancer. Gut 2007; 56: 1426-1432
[28] Hanrahan V, Currie M], Gunningham SP et al. The angiogenic switch for vascular endothelial growth factor (VEGF)-A, VEGF-B, VEGF-C, and VEGF-D in the adenoma-carcinoma sequence during colorectal cancer progression. J Pathol 2003; 200: 183-194

[29] Tjalma JJ, Garcia-Allende PB, Hartmans E et al. Molecular fluorescence endoscopy targeting vascular endothelial growth factor A for improved colorectal polyp detection. J Nucl Med 2016; 57: 480 - 485 